\title{
Ridge waveguide InGaN/GaN quantum dot edge emitting visible lasers
}

\section{Animesh Banerjee, Thomas Frost ${ }^{*}$, Shafat Jahangir, Ethan Stark, and Pallab Bhattacharya}

Department of Electrical Engineering and Computer Science, Center for Photonics and Multiscale Nanomaterials, University of Michigan, Ann Arbor, Michigan 48109-2122, USA

Received 14 August 2012, revised 20 November 2012, accepted 27 November 2012

Published online 1 February 2013

Keywords quantum dot, molecular beam epitaxy, visible lasers, c-plane $\mathrm{GaN}$

*Corresponding author: e-mail tfrost@umich.edu

Blue-emitting and green-emitting laser heterostructures were grown by molecular beam epitaxy, incorporating $\mathrm{InGaN} / \mathrm{GaN}$ quantum dots as the active medium, with a measured quantum efficiency of $60 \%$ on $n-G a N$ bulk substrates. These quantum dots exhibit no S-shape in the photoluminescence peak wavelength as a function of temperature, as typically found in comparable quantum well devices. The lasers were characterized by a threshold current density, $\mathrm{J}_{\mathrm{th}}$, of $930 \mathrm{~A} / \mathrm{cm}^{2}$ under pulsed bias, with a differential efficiency of $13.9 \%$, and a wall plug (power conversion) efficiency of $0.4 \%$ at $1050 \mathrm{~A} / \mathrm{cm}^{2}$. Green-emitting quantum dot lasers have $J_{\text {th }}=935 \mathrm{~A} / \mathrm{cm}^{2}$. The lasers were also characterized by their modal properties through near field imaging.
1 Introduction Solid state lasers, operating in the visible wavelength range, are necessary for several applications including for use in optical data storage, solid state lighting, heads-up displays, and full-color mobile projectors $[1,2]$. For blue and green wavelength devices, the laser heterostructure typically incorporates multiple InGaN/GaN quantum wells (MQW) in the gain region of the laser $[3,4]$ Due to the large polarization field present in devices grown on c-plane GaN, lasers grown on this orientation incorporating quantum wells suffer from reduced electron-hole overlap and consequently reduced gain and increased threshold. To reduce the polarization field and related effects, lasers have recently been grown on semi-polar and non-polar substrates $[5,6]$, along with novel quantum well designs for larger electron hole overlap [7, 8]. However, difficulty in incorporating In in InGaN/GaN QWs on these substrates can impede the growth of such lasers [9]. As an alternative, quantum dots, which form due to strain relaxation, have been shown theoretically to have a reduced polarization field [10], and consequently increased carrier confinement, smaller quantum confined Stark effect (QCSE), reduced blue-shift of the emission peak, reduced carrier recombination lifetimes, and a smaller rate of recombination at defects $[11,12]$. Such lasers are typically characterized by their threshold current, differential efficiency, and modal characteristics. Hence it is important to design structures to optimize these parameters, and to characterize such devices accordingly. We recently demonstrated a green $\mathrm{InGaN} / \mathrm{GaN}$ quantum dot laser $(\lambda=524)$ with low threshold current density [13]. Here, we discuss the growth of blue InGaN/GaN quantum dots and lasers incorporating such quantum dots into the active region. Optimized blue quantum dots with an efficiency of $\sim 60 \%$ are demonstrated. The lasers incorporating these quantum dots are characterized by a threshold current density of 930 $\mathrm{A} / \mathrm{cm}^{2}$ and a differential efficiency of $13.9 \%$. Lasers with a $2 \mu \mathrm{m}$ ridge width were characterized by a single mode in the growth direction, and multimode in the lateral direction, indicating a smaller ridge width is necessary to obtain single mode operation. Preliminary results on green-emitting lasers are also presented

2 Experiment The laser heterostructures, as shown in Fig. 1(a) were grown by a plasma-assisted Veeco Gen II molecular beam epitaxy (PA-MBE) system equipped with standard $\mathrm{Ga}$, In, $\mathrm{Al}, \mathrm{Si}$, and $\mathrm{Mg}$ effusion cells, and a UNIbulb nitrogen plasma source. The samples were grown on c-plane $\mathrm{n}-\mathrm{GaN}$ bulk substrates having a low defect density of $\sim 5 \times 10^{6} \mathrm{~cm}^{-2}$. The growth temperature was measured by an infrared pyrometer, calibrated by the reflection high energy electron diffraction (RHEED) transition of $7 \mathrm{x} 7$ to $1 \mathrm{x} 1$ of $\mathrm{Si}(111)$ substrates. $500 \mathrm{~nm}$ of molybdenum was deposited on the backside of the sample to assist in calibration of growth temperature using radiative heating.

An underlying, $500 \mathrm{~nm}$ thick, n-doped $\left(5 \times 10^{18} \mathrm{~cm}^{-3}\right)$ GaN buffer layer was grown on commercial HVPE grown c-plane n-GaN bulk substrates. The substrates were first cleaned using standard solvents, followed by a two-step 
thermal degassing to remove surface contamination at 200 ${ }^{\circ} \mathrm{C}$ for $1 \mathrm{hr}$ and at $400{ }^{\circ} \mathrm{C}$ for 40 minutes under high vacuum. The GaN was grown at $740{ }^{\circ} \mathrm{C}$, at a flux of $\Phi_{\mathrm{Ga}}=4.5$ $\mathrm{nm} / \mathrm{min}$ during which the RHEED pattern remained bright and streaky. Following the growth of the buffer layer, 700 $\mathrm{nm}$ of $\mathrm{Al}_{0.07} \mathrm{Ga}_{0.93} \mathrm{~N}$ cladding and $150 \mathrm{~nm}$ of $\mathrm{In}_{0.05} \mathrm{Ga}_{0.95} \mathrm{~N}$ waveguide layers were grown. The cladding and waveguide layers were grown at $770{ }^{\circ} \mathrm{C}$ and $590{ }^{\circ} \mathrm{C}$, respectively, with a doping concentration of $\mathrm{n} \sim 5 \times 10^{18} \mathrm{~cm}^{-3}$ in both layers. Eight periods of $\operatorname{In}_{0.18} \mathrm{Ga}_{0.82} \mathrm{~N}$ quantum dot (QD)/GaN barrier layers were grown at a substrate temperature of $565{ }^{\circ} \mathrm{C}$ under nitrogen rich conditions at an equivalent pressure of $\Phi_{\mathrm{Ga}}: \Phi_{\mathrm{In}} \sim 2: 1$, at a growth rate of $0.5 \AA / \mathrm{s}$. Growth conditions for the quantum dots have also been optimized. Twelve monolayers of InGaN was grown, followed by a 30 seconds of nitrogen growth interruption, after which the dots were well formed giving the highest PL intensity.

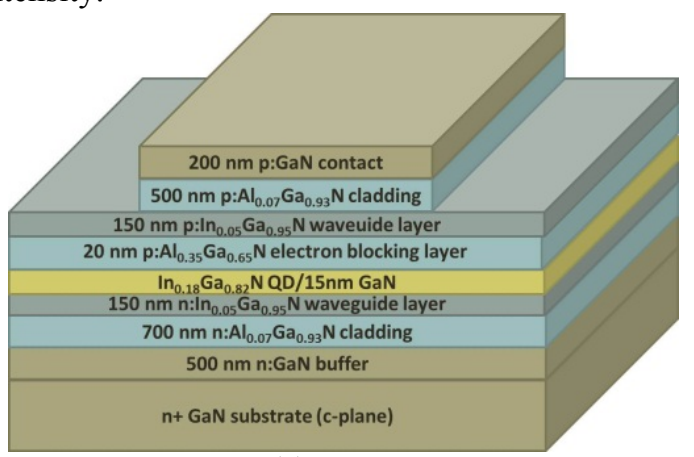

(a)

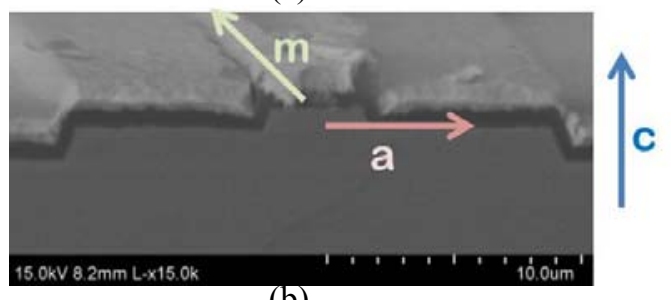

(b)

Figure 1 (a) Schematic of $\operatorname{In}_{0.18} \mathrm{Ga}_{0.82} \mathrm{~N} / \mathrm{GaN}$ QD laser heterostructure grown by plasma-assisted $\mathrm{MBE}$ showing the ridge waveguide geometry; and (b) scanning electron microscopy (SEM) image of the cleaved facet of a laser along the m-plane.

The quantum dots were capped with a $15 \mathrm{~nm}$ GaN barrier. The eight periods of quantum dots were followed by a 20 $\mathrm{nm}$ thick $\mathrm{Al}_{0.35} \mathrm{Ga}_{0.65} \mathrm{~N}$ electron blocking layer $\left(\mathrm{p} \sim 6 \mathrm{x} 10^{17}\right.$ $\mathrm{cm}^{-3}$ ), grown at $750{ }^{\circ} \mathrm{C}$. A $150 \mathrm{~nm} \mathrm{p}$-InGaN waveguide layer was grown, followed by a p-AlGaN cladding, grown at $590{ }^{\circ} \mathrm{C}$ and $770{ }^{\circ} \mathrm{C}$, respectively, with doping concentrations of $5 \times 10^{17} \mathrm{~cm}^{-3}$ and $2 \times 10^{17} \mathrm{~cm}^{-3}$, respectively. Finally, a $\mathrm{p}$-doped GaN layer was grown $\left(\mathrm{p} \sim 7 \times 10^{17} \mathrm{~cm}^{-3}\right)$ as the topmost layer for injections of holes.

The quantum dot layers in the active region were grown to have strong room temperature emission at 423 $\mathrm{nm}$, as shown in Fig. 2(a). In the inset to Fig. 2(a), an atomic force microscopy (AFM) image of quantum dots, with each quantum dot having an average height of $\sim 3 \mathrm{~nm}$ and base width of $\sim 30 \mathrm{~nm}$, is shown. From the AFM measurements, quantum dot densities of $\sim 4 \times 10^{10} \mathrm{~cm}^{-2}$ and $8 \times 10^{10} \mathrm{~cm}^{-2}$ are estimated for the green and blue emitting devices, respectively. Temperature dependent photoluminescence measurements on eight layers of InGaN/GaN quantum dot layers yield an efficiency of $\sim 60 \%$, assuming all non-radiative centers are frozen at low temperatures (Fig. 2(b)). Time resolved photoluminescence (TRPL) measurements (at room temperature) were also done yielding a mono-exponential decay from which a total carrier lifetime of $\tau=276 \mathrm{ps}$ is derived. The peak wavelength shift of the quantum dot emission with temperature (Fig. 2(c)) shows an absence of S-shaped behavior commonly seen in InGaN-based quantum wells [14]. This indicates absence of any indium clustering within the dots. The measured wavelength shift fits well with the plot of the Varshni relation (solid line in Fig. 2(c)) showing temperaturedependent energy band-gap shift given by:

$$
E_{g}(T)=E_{g}(0)-\frac{\alpha T^{2}}{T+\beta}
$$

where $\alpha=0.86 \mathrm{meV} / \mathrm{K}$ and $\beta=793 \mathrm{~K}$.

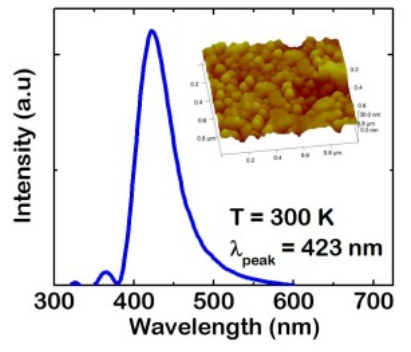

(a)

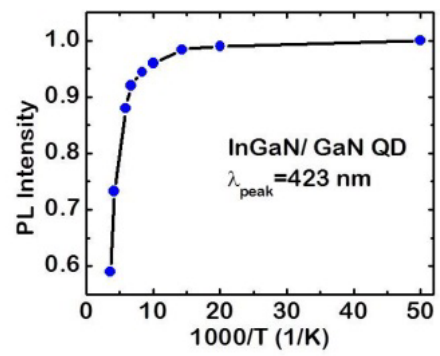

(b)

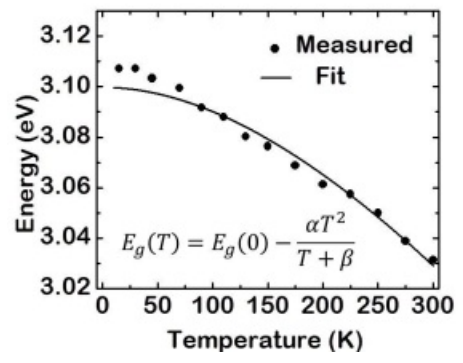

(c)

Figure 2 (a) Room temperature photoluminescence spectrum of eight pairs of $\mathrm{In}_{0.18} \mathrm{Ga}_{0.82} \mathrm{~N} / \mathrm{GaN}$ QDs with an AFM of a QD layer inset; (b) plot of integrated PL intensities with inverse temperatures, and (c) peak wavelength shift of QDs with temperature showing good fit with the Varshni relation.

Ridge waveguide lasers were fabricated from the grown laser heterostructures using standard photolithography, reactive ion etching (RIE), and contact metallization techniques. The ridge was etched to the upper AlGaN cladding/InGaN waveguide heterointerface in order to 
maximize the optical confinement, while minimizing the losses associated with the etched heterostructure/air interface. The facets were cleaved along m-plane with the laser cavity along a-plane as shown in Fig. 1(b). High reflectivity dielectric facet coatings were applied to the two facets, having reflectivities of $65 \%$ and $90 \%$ by depositing 3 and 5 pairs of $\mathrm{SiO}_{2} / \mathrm{TiO}_{2}$ DBRs, respectively.

3 Results and discussion The lasers were characterized by light-current characteristics under both pulsed and continuous wave $(\mathrm{CW})$ bias. Data for a $2 \mu \mathrm{m} \times 1 \mathrm{~mm}$ ridge waveguide device are shown in Fig. 3(a). The threshold current density for the device is $\mathrm{J}_{\mathrm{th}}=930$ and $970 \mathrm{~A} / \mathrm{cm}^{2}$ for pulsed and CW bias, respectively. These values are significantly lower than those typically found in similar quantum well lasers. Under pulsed bias, this device was characterized by a slope efficiency of $0.41 \mathrm{~W} / \mathrm{A}$, corresponding to a differential quantum efficiency of $13.9 \%$. At a bias of $1050 \mathrm{~A} / \mathrm{cm}^{2}$, the wall plug efficiency is $0.4 \%$. Figure 3(b) shows the measured lasing spectrum in comparison with the PL spectrum from the quantum dots. From the lasing and PL spectra, a peak blue-shift of only $4.4 \mathrm{~nm}$ is observed. InGaN self-organized QDs grow in StanskiKrastanow growth mode and are formed due to strain relaxation of the lattice mismatched $\mathrm{InGaN}$ layer on $\mathrm{GaN}$. Theoretical calculations [10] have also shown a much reduced polarization field present in the InGaN QDs. Assuming an effective height for QDs, from the peak emission shift, a polarization field of $\sim 80 \mathrm{kV} / \mathrm{cm}$ is calculated for the QDs which matches well with the theory, and is much lower compared with similar quantum well devices $(\sim 2.1$ $\mathrm{MV} / \mathrm{cm})[15,16]$.

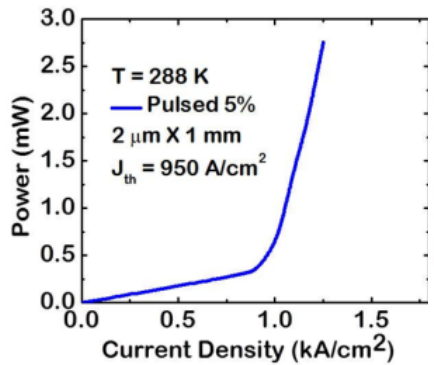

(a)

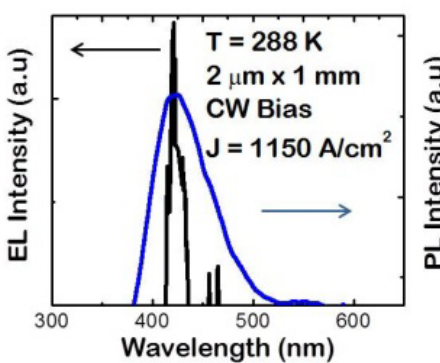

(b)
Figure 3 (a) Light-current characteristics of blue-emitting $\mathrm{In}_{0.18} \mathrm{Ga}_{0.82} \mathrm{~N} / \mathrm{GaN}$ QD ridge waveguide laser measured under pulsed bias; (b) electroluminescence spectra of QD laser above threshold along with PL of $\operatorname{In}_{0.18} \mathrm{Ga}_{0.82} \mathrm{~N}$ QDs.

A near field image from a $4 \mu \mathrm{m}$ wide ridge waveguide laser, with a cavity length of $1.2 \mathrm{~mm}$, is shown as a contour plot in Fig. 4(a). The measured mode profile in the growth (transverse) direction, intersecting the maximum of the mode, is shown in Fig. 4(b), with the mode in the direction perpendicular (lateral) direction (along the width of the ridge) shown in Fig. 4(c). In the growth direction, the relatively narrow thickness allows for a (nearly) single mode output, while the mode in the transverse direction clearly consists of several higher order modes, as expected in such a relatively wide waveguide. Additionally the waveguide modes were calculated by transfer matrix method in the transverse and lateral directions, and plotted alongside the measured modes in the respective figures. The mode in the lateral direction is fit well by just the fundamental mode in that direction. The mode in the lateral direction is fit as a linear combination of the three calculated waveguide modes in order to best match the core of the waveguide. While the calculated mode in the transverse direction closely matches the simulated mode, the mode in the lateral direction shows a reduction in confinement as compared with the simulated mode. This is likely due to the ridge being etched less than the design.

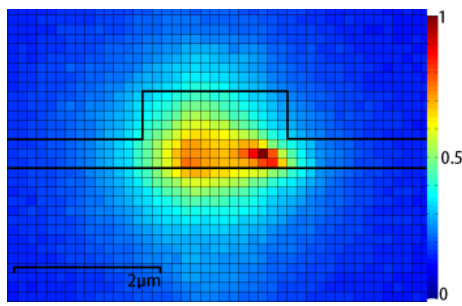

(a)

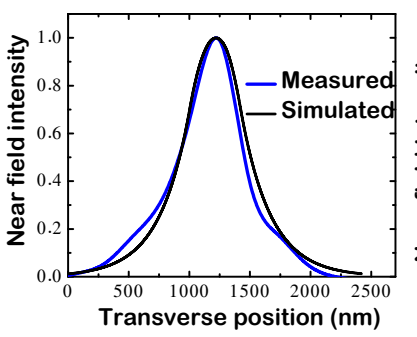

(b)

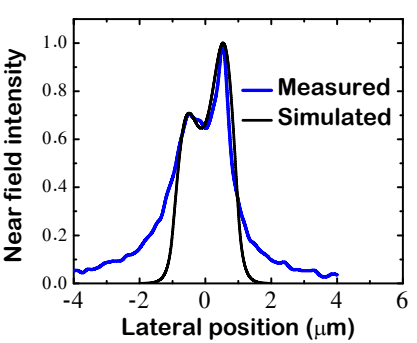

(c)
Figure 4 (a) Contour plot of the near field image of the laser mode; laser mode profile in the transverse (b) and longitudinal (c) directions, as compared with the mode profiles simulated by transfer matrix method.

Furthermore, light-current characteristics were taken for devices of varying lengths, from 0.6 to $1.6 \mathrm{~mm}$. From analysis of the differential efficiency with cavity length, a value of internal quantum efficiency of $66 \%$ is calculated, with an internal (cavity) loss of $11 \mathrm{~cm}^{-1}$. From the variation in threshold current density with cavity length, a value of differential gain of $\mathrm{dg} / \mathrm{dn}=2 \times 10^{-16} \mathrm{~cm}^{2}$ is derived. This is in good agreement with our previously calculated value for differential gain in these InGaN quantum dots lasers, and an order of magnitude higher than comparable quantum well lasers $[17,18]$.

Additionally, laser heterostructures incorporating $\mathrm{In}_{0.32} \mathrm{Ga}_{0.68} \mathrm{~N} / \mathrm{GaN}$ green emitting quantum dots $(\lambda=560$ $\mathrm{nm})$ were also grown and characterized. Under pulsed bias, a lasing threshold of $935 \mathrm{~A} / \mathrm{cm}^{2}$ is observed, as shown in Fig. 5 from a ridge waveguide device with a cavity length and width of $0.85 \mathrm{~mm}$ and $5 \mu \mathrm{m}$, respectively. A slope efficiency of $0.25 \mathrm{~W} / \mathrm{A}$ is observed, corresponding to a differential quantum efficiency of $11.3 \%$. 


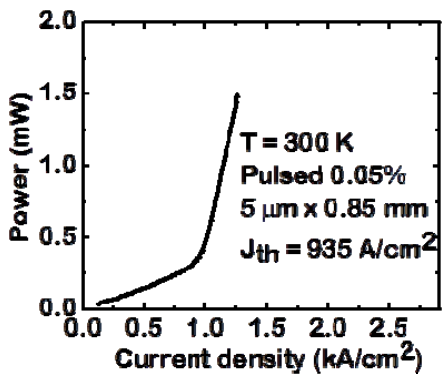

Figure 5 Light-current characteristics of green emitting $\mathrm{In}_{0.32} \mathrm{Ga}_{0.68} \mathrm{~N} / \mathrm{GaN}$ QD ridge waveguide laser measured under pulsed bias current.

4 Conclusion The optimized growth parameters of self-organized $\operatorname{In}_{0.18} \mathrm{Ga}_{0.82} \mathrm{~N} / \mathrm{GaN}$ QDs have been obtained and blue-emitting ridge waveguide lasers incorporating the optimized QDs have been fabricated and measured. The QDs are found to have a high efficiency of $\sim 60 \%$ with no S-shaped behavior in their peak wavelength shift with temperature as generally found in InGaN quantum wells. The lasers have been operated under pulsed and $\mathrm{CW}$ bias conditions. A threshold current of $930 \mathrm{~A} / \mathrm{cm}^{2}$ is measured in a $2 \mu \mathrm{m}$ ridge laser at $288 \mathrm{~K}$ under pulsed bias in the blue emitting lasers. In the green-emitting lasers, a threshold current density of $935 \mathrm{~A} / \mathrm{cm}^{2}$ was measured under pulsed bias in a $5 \mu \mathrm{m}$ ridge laser. The blue shift due to screening of the piezoelectric field in the dots is $4.4 \mathrm{~nm}$. Near-field imaging from the ridge waveguide lasers show single mode output in the transverse and multi-mode output in the lateral directions that fit well with the simulated waveguide modes.

Acknowledgements The work has been supported by the National Science Foundation (MRSEC program) under Grant 0968346.

\section{References}

[1] H. Ohta, S. P. DenBaars, and S. Nakamura, J. Opt. Soc. Am. B 27, B45 (2010).

[2] O. H. Nam, K. H. Ha, J. S. Kwak, S. N. Lee, K. K. Choi, T. H. Chang, S. H. Chae, W. S. Lee, Y. J. Sung, H. S. Paek, J. H. Chae, T. Sakong, J. K. Son, H. Y. Ryu, Y. H. Kim, and Y. Park, Phys. Status Solidi A 201(12), 2712 (2004).

[3] S. Nakamura, M. Senoh, S. Nagahama, N. Iwasa, and T. Yamada, Appl. Phys. Lett. 69, 4056 (1996).

[4] K. Okamoto, J. Kashiwagi, T. Tanaka, and M. Kubota, Appl. Phys. Lett. 94, 071105 (2009).

[5] R. M. Farrell, E. C. Young, F. Wu, S. P. DenBaars, and J. S. Speck, Semicond. Sci. Technol. 27, 024001 (2012).

[6] P. S. Hsu, M. T. Hardy, F. Wu, I. Koslow, E. C. Young, A. E. Romanov, K. Fujito, D. Feezell, S. P. DenBaars, J. S. Speck, and S. Nakamura, Appl. Phys. Lett. 100, 0211104 (2012).

[7] H. Zhao, G. Liu, J. Zhang, J. D. Poplawsky, V. Dierolf, and N. Tansu, Opt. Express 19(54), A991 (2011).

[8] H. Zhao and N. Tansu, J. Appl. Phys. 107, 113110 (2012).

[9] F. Wu, Y.-D. Lin, A. Chakraborty, H. Ohta, S. P. DenBaars, S. Nakamura, and J. S. Speck, Appl. Phys. Lett. 96, 231912 (2010).

[10] Y.-R. Wu, Y.-Y. Lin, H.-H. Huang, and J. Singh, J. Appl. Phys. 105, 013117 (2009).

[11] S. Schulz and E. P. O' Reilly, Phys. Rev. B 82, 033411 (2010).

[12] M. Zhang, P. Bhattacharya, and W. Guo, Appl. Phys. Lett. 97, 011103 (2010).

[13] M. Zhang, A. Banerjee, C.-S. Lee, J. M. Hinckley, and P. Bhattacharya, Appl. Phys. Lett. 98, 221104 (2011).

[14] Y.-H. Cho, G. H. Gainer, A. J. Fischer, J. J. Song, S. Keller, U. K. Mishra, and S. P. DenBaars, Appl. Phys. Lett. 73(10), 1370 (1998).

[15] J. Müller, G. Brüderl, M. Schillgalies, S. Tautz, D. Dini, A. Breidenassel, B. Galler, and S. Lutgen, Appl. Phys. Lett. 95, 051104 (2009).

[16] Y. D. Jho, J. S. Yahng, E. Oh, and D. S. Kim, Phys. Rev. B 66, 035334 (2002).

[17] H. Zhao, R. A. Arif, Y. K. Ee, and N. Tansu, Opt. Quantum Electron. 40, 301 (2008).

[18] W. G. Scheibenzuber, U. T. Schwarz, L. Sulmoni, J. Dorsaz, J.-F. Carlin, and N. Grandjean, J. Appl. Phys. 109, 093106 (2011). 